\title{
(GIGA)bYte
}

\section{Chromosome-level assembly of the common vetch (Vicia sativa) reference genome}

\author{
Hangwei Xii ${ }^{1}$, Vy Nguyen ${ }^{1}$, Christopher Ward ${ }^{1}$, Zhipeng Liu $^{2, *}$ and \\ Iain R. Searle ${ }^{1, *}$
}

1 School of Biological Sciences, The University of Adelaide, Adelaide, Adelaide 5005, Australia

2 State Key Laboratory of Grassland Agro-ecosystems, College of Pastoral Agriculture Science and Technology, Lanzhou University, No 768 Jiayuguan West Road, Chengguan District, Lanzhou 730020, China

\section{ABSTRACT}

Vicia sativa L. (common vetch, $n=6$ ) is an annual, herbaceous, climbing legume, originating in the Fertile Crescent of the Middle East and now widespread in the Mediterranean basin, West, Central and Eastern Asia, North and South America. V. sativa is of economic importance as a forage legume in countries such as Australia, China, and the USA, and contributes valuable nitrogen to agricultural rotation cropping systems. To accelerate precision genome breeding and genomics-based selection of this legume, we present a chromosome-level reference genome sequence for $V$. sativa, constructed using a combination of long-read Oxford Nanopore sequencing, short-read Illumina sequencing, and high-throughput chromosome conformation data (CHiCAGO and Hi-C) analysis. The chromosome-level assembly of six pseudo-chromosomes has a total genome length of $1.65 \mathrm{Gbp}$, with a median contig length of $684 \mathrm{Kbp}$. BUSCO analysis of the assembly demonstrated very high completeness of $98 \%$ of the dicotyledonous orthologs. RNA-seq analysis and gene modelling enabled the annotation of 53,218 protein-coding genes. This $V$. sativa assembly will provide insights into vetch genome evolution and be a valuable resource for genomic breeding, genetic diversity and for understanding adaption to diverse arid environments.

Subjects Genetics and Genomics, Bioinformatics, Plant Genetics

Submitted: 17 October 2021 Accepted: $\quad 26$ January 2022

Published: $\quad 31$ January 2022

* Corresponding authors. E-mail: lzp@lzu.edu.cn;

iain.searle@adelaide.edu.au

Published by GigaScience Press.

Preprint submitted at https: //doi.org/10.1101/2021.10.11.464017

This is an Open Access article distributed under the terms of the Creative Commons Attribution License (http://creativecommons.org/ licenses/by/4.0/), which permits unrestricted reuse, distribution, and reproduction in any medium, provided the original work is properly cited.

Gigabyte, 2022, 1-19

\section{DATA DESCRIPTION}

\section{Background}

Vicia sativa L. (common vetch, NCBI:txid3908) (Figure 1) is an annual legume belonging to the Fabaceae family, and Vicia genus [1]. The Vicia genus contains about 180-210 species, including the economically important crop broad bean [2]. To date, no chromosome-level genome assembly has been reported within the Vicia genus. Interestingly, V. sativa has at least three different reported haploid chromosome numbers: $n=5,6$ or 7 [3], but $n=6$ is the best characterized karyotype.

$V$. sativa is thought to have originated in the Fertile Crescent of the Middle East and is now widespread on every continent as both a crop and a weed [4]. V. sativa is a multipurpose legume; the plants are often grown for forage and the seeds can be used safely as a feed for ruminant animals. V. sativa seed contains up to $30 \%$ crude protein and is rich in essential amino acids and unsaturated fatty acids [5]. However, only a small amount of the seed can be safely fed to monogastric animals like chickens and pigs, because of the 

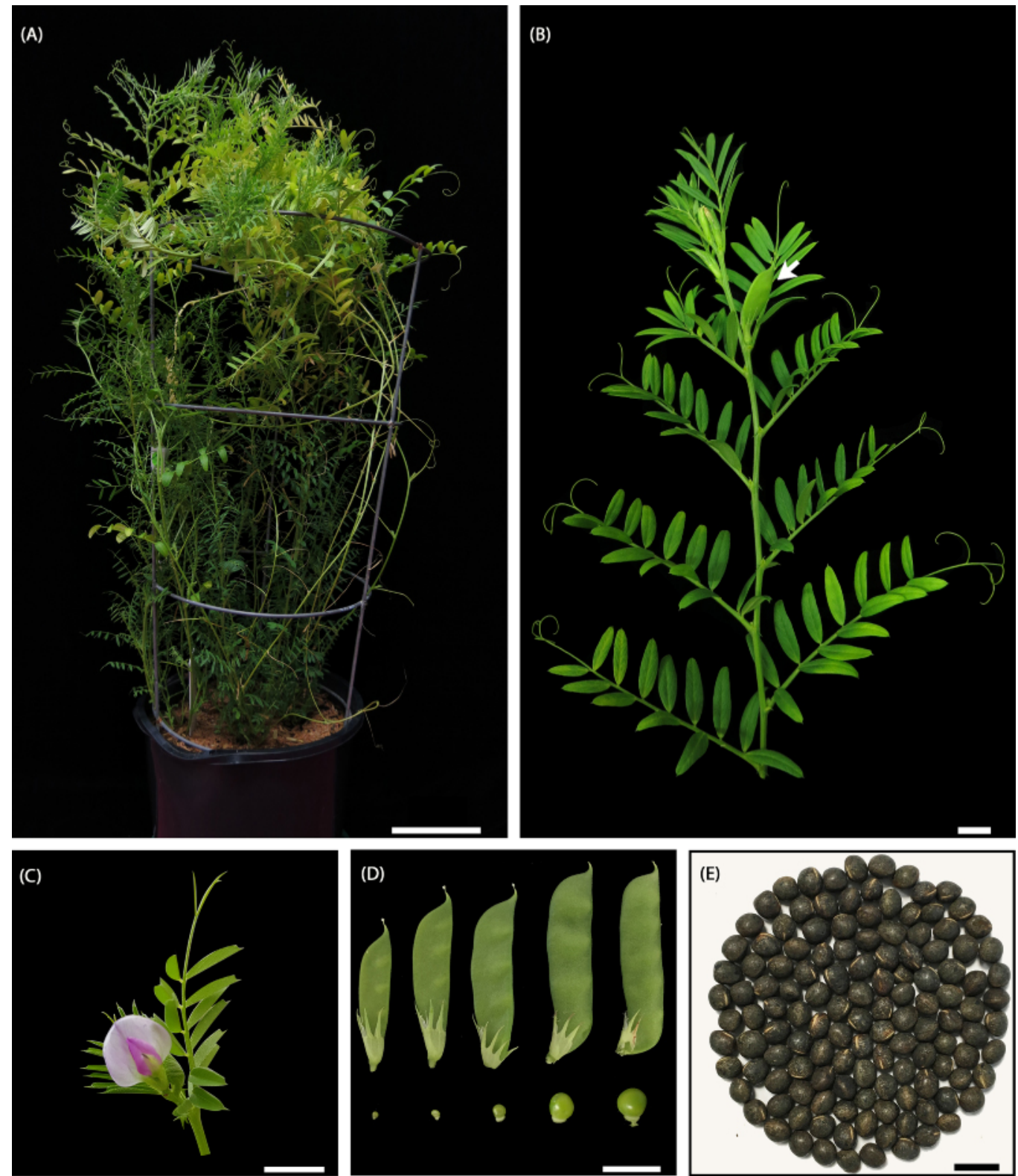

Figure 1. Vicia sativa (cultivar Studenica). (A) Ten-week-old V. sativa at flowering. (B) Detached stem showing compound leaves at each node. At the end of each compound leaf is a tendril. A single pod forms at the base of each leaf node after flowering (arrow). (C) A shoot apex with a flower and surrounding young leaves. (D) Young to mature seed pods (left to right), with a representative seed shown at the bottom of each pod. Each pod contains 3-5 seeds. (E) Dry seeds of cultivar Studenica. Scale bars $(A)=10 \mathrm{~cm},(B, C, E)=1 \mathrm{~cm},(D)=2 \mathrm{~cm}$.

presence of the neurotoxic proteinaceous amino acids $\beta$-cyano-L-alanine and $\gamma$-glutamyl- $\beta$-cyano-alanine [6].

$V$. sativa is often used in crop rotation systems to increase nitrogen input to the soil. In a study of $V$. sativa/wheat rotation over a 4-year-period, cultivation of $V$. sativa during autumn increased soil water storage and subsequently increased biological yield and grain yield of wheat. Both yields were doubled in the third year compared with the second year of the rotation [7]. Furthermore, the symbiosis between soil rhizobia bacteria and $V$. sativa 
roots allows the plant to fix atmospheric nitrogen and later provide nitrogen for the following crop, hence reducing the use of expensive nitrogen fertilizer [8]. V. sativa exhibits excellent drought tolerance and is suitable for cultivation in arid areas. In one drought tolerance study, $V$. sativa could withstand a month of drought stress, with the leaf weight not decreasing significantly compared with the non-drought control [9]. V. sativa offers multiple usage and is a valuable crop in a sustainable agricultural system [10].

With the important value of $V$. sativa, vetch breeders have primarily selected for traits conferring high yield, pod shattering, flowering time, disease resistance against Ascochyta fabae, Uromyces viciae-fabae (rust) and Sclerotinia sclerotium [11]. Recently published transcriptome data has allowed agriculturally important traits to be uncovered at the gene expression level, such as pod-shattering resistance [12] and drought tolerance genes [13] in $V$. sativa. However, a lack of high-quality genome reference is currently impeding the genetic mapping of important genes and hindering further applications such as genome editing when compared with other crops.

\section{Context}

In this study, we assembled a high-quality chromosome-level reference genome for $V$. sativa, which is the first chromosome-level reference genome in the Vicia genus. We performed genome annotation using RNA-seq data from five tissues to ensure most of the expressed genes were captured. We also included a phylogenetic analysis of $V$. sativa and legume relatives. We envisage that our $V$. sativa genome will be an important resource for evolutionary studies of this species. The well-annotated chromosome-level genome will also provide important information to facilitate genetic mapping, gene discovery and functional gene studies.

\section{METHODS}

\section{Sampling and sequencing}

To prepare V. sativa for whole genome sequencing (WGS) using long-read and short-read data, seeds of cultivar Studenica ( $V$. sativa subsp. sativa) were obtained from the South Australian Research and Development Institute (SARDI, South Australia, Australia). Seeds were sterilized and germinated in vitro on half-strength Murashige \& Skoog (1/2 MS) basal medium with $1 \%$ sucrose for 3 days at $25^{\circ} \mathrm{C}$, in the dark. Bulk 3-mm-long primary root tips were then harvested and snap-frozen in liquid nitrogen for subsequent DNA extraction. DNA was extracted using the phenol:chloroform method [14], with an additional high-salt low-ethanol wash to improve DNA purity [15]. High-quality DNA was confirmed by electrophoresis on 1\% agarose gel. The DNA was sent to the Australian Genome Research Facility (AGRF, Melbourne, Australia), and Novogene Co., Ltd (Hong Kong, China) for library preparation and sequencing on a PromethION (PromethION, RRID:SCR_017987) and Novo-Seq 6000 (Illumina NovaSeq 6000 Sequencing System, RRID:SCR_016387), respectively. We obtained 72 gigabase pairs (Gbp) of Nanopore long-read data, and $205 \mathrm{~Gb}$ paired-end short-read data (150 base pairs [bp] read length).

To produce $V$. sativa CHiCAGO sequencing data [16] and Hi-C sequencing data [17], $2 \mathrm{~g}$ of young leaf tissue was snap-frozen in liquid nitrogen and sent to Dovetail Genomics (USA) for library preparation and sequencing. CHiCAGO and Hi-C libraries were sequenced on an Illumina HiSeq X (Illumina HiSeq X Ten, RRID:SCR_016385) to produce 162 Gbp of CHiCAGO and $148 \mathrm{Gbp}$ of Hi-C sequencing data, respectively. 


\begin{tabular}{|c|c|c|c|c|c|}
\hline Libraries & Insert size (bp) & Raw data (Gbp) & Clean data (Gbp) & $\begin{array}{l}\text { Mean read } \\
\text { length (bp) }\end{array}$ & Coverage $(x)^{*}$ \\
\hline $\begin{array}{l}\text { WGS Illumina } \\
\text { short-reads }\end{array}$ & 300 & 205.13 & 200.28 & 150 & 124.32 \\
\hline Nanopore reads & N/A & 72.12 & N/A & 9094 & 43.71 \\
\hline CHiCAGO & 350 & 162.00 & N/A & 150 & 98.18 \\
\hline $\mathrm{Hi}-\mathrm{C}$ & 350 & 147.60 & N/A & 150 & 89.45 \\
\hline $\begin{array}{l}\text { Illumina RNA-seq } \\
\text { reads }\end{array}$ & 300 & 74.60 & 66.49 & 150 & 45.21 \\
\hline
\end{tabular}

To prepare $V$. sativa RNA sequencing (RNA-seq) data, RNA was purified from the first two fully expanded leaves, shoot apexes with young leaves up to $1 \mathrm{~cm}$ long from 4-week-old plants, roots from 5-day-old seedlings and 4-week-old leaf-derived callus tissues using the Spectrum $^{\text {TM }}$ Plant Total RNA Kit (Sigma Aldrich). Additional DNase I treatment was used to remove DNA contamination (On-Column DNase I Digestion, Sigma Aldrich), and ribosome removal treatment to enrich for the non-ribosomal RNA fraction (Ribo-Zero rRNA Removal Kit for Plant Leaf or Plant Seed/Root, Illumina) [18]. Directional RNA libraries were prepared for each tissue using the NEBNext Ultra ${ }^{\mathrm{TM}}$ Directional RNA Library Prep Kit for Illumina (New England Biolabs) following the manufacturer's protocol. Libraries were sent to Novogene Co., Ltd (Hong Kong, China) for sequencing on Novo-Seq 6000 (Illumina) to obtain 150-bp paired-end read data. In total, we obtained $74.6 \mathrm{Gbp}$ of RNA-seq data. A summary of the long and short-read sequencing data is provided in Table 1.

\section{Genome size estimation and genome assembly}

We first performed a genome size estimation for $V$. sativa. To do this, short-Illumina (paired-end $150 \mathrm{nt}$ ) reads were trimmed using TrimGalore v0.4.2 (Trim Galore, RRID:SCR_011847) with default parameters and 25-mers were counted using Jellyfish v2.2.6 (Jellyfish, RRID:SCR_005491) [19]. The 25-mer count distribution data was used as an input to GenomeScope (GenomeScope, RRID:SCR_017014) [20] for genome size estimation with the read length set to 150 and max $k$-mer coverage set to 1 million. GenomeScope estimated a genome-wide heterozygosity level of $0.09 \%$ (Figure 2) and a genome size of $1.61 \mathrm{Gbp}$; approximately 160 megabase pairs (Mbp) smaller than the genome size estimated by flow cytometry (1.77 Gbp) [21].

Next, we conducted contig assembly from the Nanopore long-reads using Canu v1.7 (Canu, RRID:SCR_015880) [22] under default parameters with the expected genome size set at $1.77 \mathrm{Gbp}$. Canu was used to perform read trimming and sequencing error correction for the input data before performing contig assembly. The assembled contigs were polished using clean WGS short-reads with Pilon v1.22 (Pilon, RRID:SCR_014731) [23] under default parameters. We repeated the polishing step and observed a further improvement in contig quality (Table 2). Contig quality was assessed using BUSCO v5.2.2 (BUSCO,

RRID:SCR_015008) [24] for the completeness of the genome, and after two rounds of polishing, complete BUSCOs increased from $69.9 \%$ to $97.8 \%$ (Table 2). Overall, we obtained 9,990 assembled contigs, which were $1.93 \mathrm{Gbp}$, with an N50 value of 685 kilobase pairs (Kbp). 


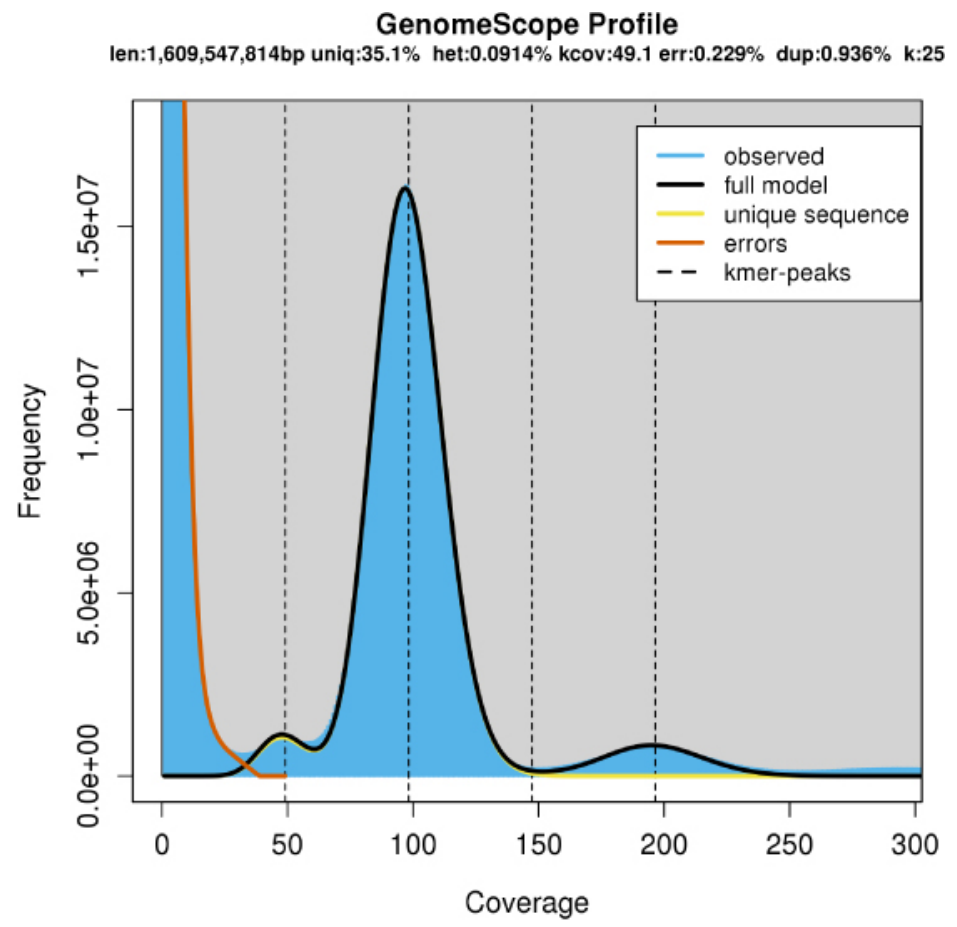

Figure 2. 25-mer distribution of Illumina paired-end reads by using GenomeScope. 25-mer occurrences ( $x$ axis) were plotted against their frequencies ( $y$ axis). Estimated genome size: 1,609,547,814 bp, estimated unique region: $35.1 \%$, estimated heterozygosity: $0.0914 \%$, estimated genome.

Table 2. Genome completeness evaluated by BUSCO with fabales_odb10 dataset (number of BUSCOs: 5366) after the first and second round of polishing $V$. sativa contigs using WGS short-read data.

\begin{tabular}{|c|c|c|c|}
\hline BUSCO analysis & No polishing (\%) & $1^{\text {st }}$ polishing (\%) & $2^{\text {nd }}$ polishing (\%) \\
\hline Complete & 69.9 & 97.7 & 97.8 \\
\hline Complete and single-copy & 63 & 87.3 & 88.9 \\
\hline Complete and duplicated & 6.9 & 10.4 & 8.9 \\
\hline Fragmented & 3.5 & 0.3 & 0.3 \\
\hline Missing & 26.6 & 2.0 & 1.9 \\
\hline
\end{tabular}

\section{Chromosome-level assembly using Hi-C and linkage map data}

To generate a chromosome-level assembly for $V$. sativa, Hi-C proximity [25] ligation data and CHiCAGO [26] were used to order and orient the contigs along chromosomes. The scaffolding process was carried out by Dovetail Genomics (Santa Cruz, CA, USA) using Dovetail ${ }^{\mathrm{TM}} \mathrm{Hi}-\mathrm{C}$ library reads to connect and order the input set of contigs. After scaffolding with HiRise (v2.1.7) [51], the assembled genome sequence initially comprised $1.8 \mathrm{Gbp}$, with a scaffold and contig N50 of 51.4 and $0.6 \mathrm{Mbp}$, respectively. A high fraction of the assembled sequences (93\%) were contained in four pseudo-chromosomes (Figure 3A); however $V$. sativa has six pairs of chromosomes [1]. We observed that two of the four pseudo-chromosomes had weak interactions, suggesting misjoining of two contigs (Figure 3A).

In parallel to the HiRise analysis, we performed a second chromosome-level assembly using 3D-DNA (3D de novo assembly, RRID:SCR_017227) [27]. 3D-DNA scaffolding was performed by first mapping Hi-C reads to the contig assembly using Juicer v1.6 
(A)

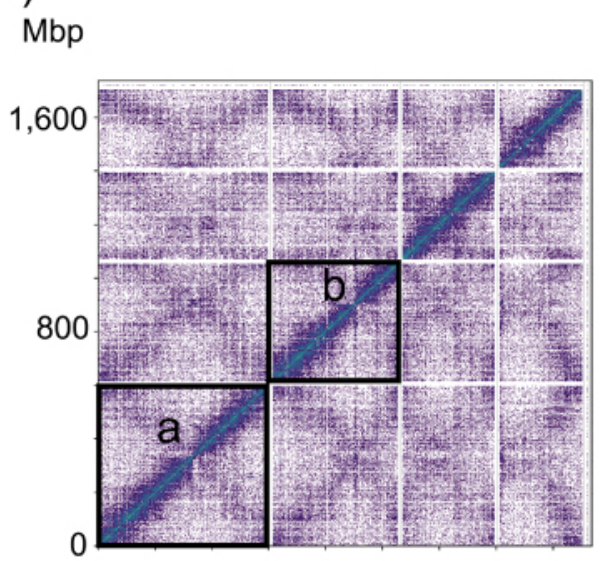

(B)

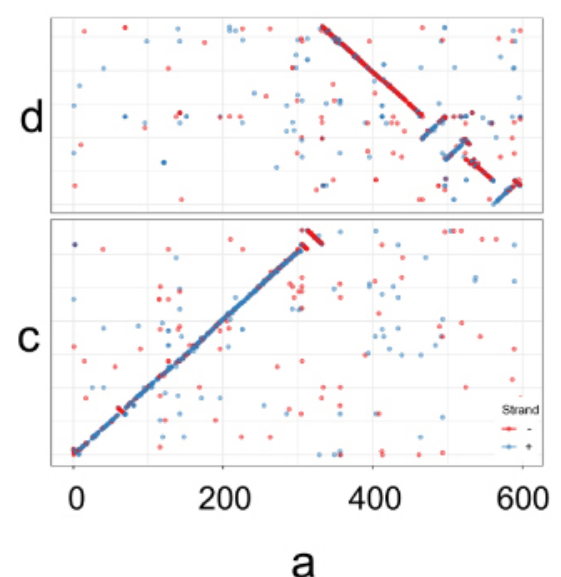

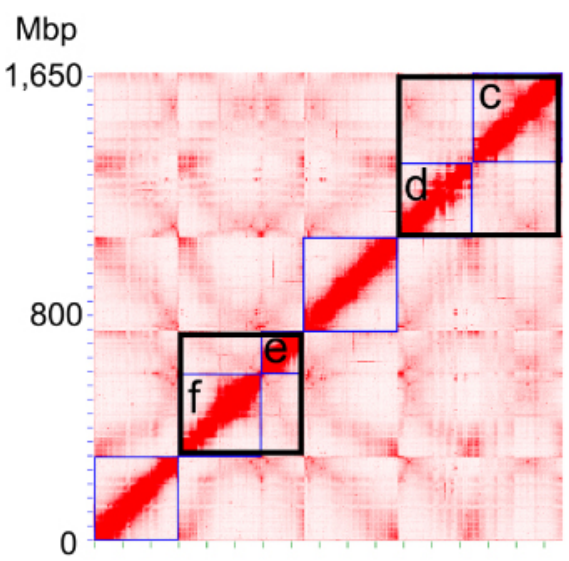

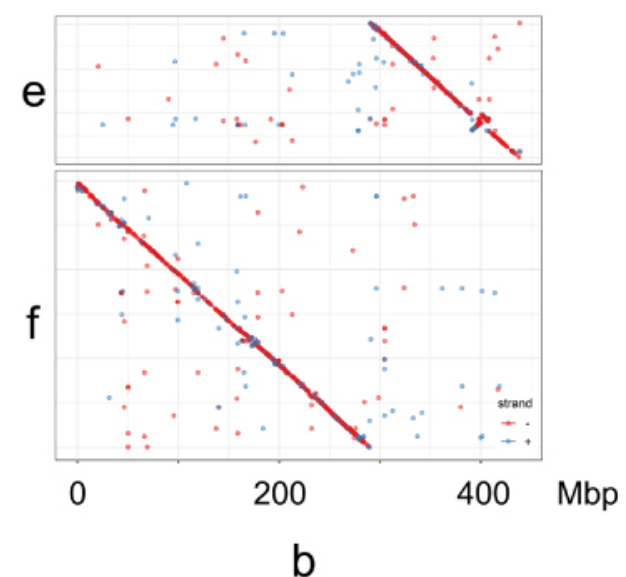

Figure 3. Resolving misjoin sites in V. sativa pseudo-chromosomes. (A) The left figure shows the interaction heatmap of four pseudo-chromosomes generated by the HiRise pipeline. Regions in black boxes show the potential misjoined pseudo-chromosomes indicated by weak interaction signals. After rescaffolding the genome using the 3D-DNA pipeline, mi-joins were confirmed and resulted in six pseudo-chromosomes (right figure, black boxes). (B) The collinearity between pseudo-chromosomes "a" to "c" and " $\mathrm{d}$ ", and between pseudo-chromosomes "b" to "e" and " $\mathrm{f}$ " in (A) were confirmed by Mummer alignment.

(Juicer, RRID:SCR_017226) [28], which then generated 304,484,352 uniquely mapped pair-end reads and of which $51.1 \%(155,477,211)$ of the uniquely mapped reads were identified as valid Hi-C contacts. The 3D-DNA v180114 pipeline was integrated to anchor contigs to pseudo-chromosomes based on valid Hi-C contacts. The output file was used to generate a Hi-C heatmap for manual inspection using Juicebox Assembly Tools v1.11.08 (Juicebox, RRID:SCR_021172). This revealed six high-quality pseudo-chromosomes (Figure 3A).

We compared the HiRise and 3D-DNA assembled pseudo-chromosomes by performing a whole genome alignment with Mummer v4.0.0 (MUMmer, RRID:SCR_018171) [29]. The alignment showed a strong synteny between the HiRise and 3D-DNA pseudo-chromosomes (Figure 3B). However, the two longest HiRise pseudo-chromosomes aligned to four 3D-DNA pseudo-chromosomes suggesting two HiRise pseudo-chromosomes were misjoined 


\begin{tabular}{|cr|}
\hline Table 3. The length of $V$. sativa pseudo-chromosomes. \\
\hline Pseudo-chromosome & Length (bp) \\
\hline 1 & $324,818,257$ \\
2 & $324,640,943$ \\
3 & $290,752,327$ \\
4 & $290,123,409$ \\
5 & $272,590,232$ \\
6 & $148,681,034$ \\
\hline Total & $1,651,606,202$ \\
\hline
\end{tabular}

\begin{tabular}{|lc|}
\hline Table 4. Overview of Vicia sativa genome assembly. & \\
\hline Feature & Value \\
Total length (bp) & $1,653,553,227$ \\
\hline No. of contigs & 9,990 \\
\hline Contig N50 length (bp) & 684,593 \\
Scaffold N50 length (bp) & $290,126,875$ \\
\hline GC content (\%) & 35.6 \\
Predicted protein-coding genes & 53,218 \\
\hline Predicted noncoding genes & 3,966 \\
Content of repetitive sequences (\%) & 83.92 \\
\hline
\end{tabular}

(Figure 3B). The putative misjoined HiRise pseudo-chromosomes also coincided with low Hi-C interactions (Figure 3A).

To further support that these two HiRise pseudo-chromosomes were misjoined, we compared the synteny of the HiRise and 3D-DNA pseudo-chromosomes to the high-quality $V$. faba genetic linkage map [30] as no genetic linkage map is available for $V$. sativa. When we compared the order of 1536 sequenced $V$. faba DNA markers to their homologous regions in our HiRise and 3D-DNA pseudo-chromosomes, we observed a clear synteny between $V$. faba and $V$. sativa. However, two out of four of the HiRise pseudo-chromosomes appeared to be misjoined, for example, the markers on HiRise pseudo-chromosomes one, mapped to two $V$. faba linkage groups (Figure 4). After combining the karyotype, Hi-C interaction and synteny data to $V$. faba, we concluded the 3D-DNA assembly was most likely correct and subsequently used this assembly in further analysis. Finally, we used purge_dup pipeline v1.2.5 (purge dups, RRID:SCR_021173) [31] to remove low coverage scaffolds, partial overlaps and haplotigs. The final version of the genome assembly contains six pseudo-chromosomes (Table 3), in which a total of $1.65 \mathrm{Gbp}$ contigs are anchored to these pseudo-chromosomes (Figure 5), and remain 10 unassigned contigs (overall 334,511 bp length). The overall genome size is $1,653,553,227 \mathrm{bp}$, with a GC content of $35.6 \%$ (Table 4).

\section{DATA VALIDATION AND QUALITY CONTROL}

Three approaches were used to assess the quality of the final version of our genome assembly. First, the WGS short-read data was mapped to this final assembly. A very high proportion (99.7\%) was mapped (Table 5). Second, the genome completeness was assessed by using BUSCO v5.2.2 referencing fabales_odb10 gene sets. Overall, BUSCO identified $97.8 \%$ complete genes (of which $8.9 \%$ were duplicated), $0.3 \%$ fragmented genes, and $1.9 \%$ missing genes out of 5366 markers in the gene sets. Finally, the LTR Assembly Index (LAI) of 12.96 was calculated by feeding the result of LTRharvest v1.6.2 (LTRharvest, 
Hi-Rise
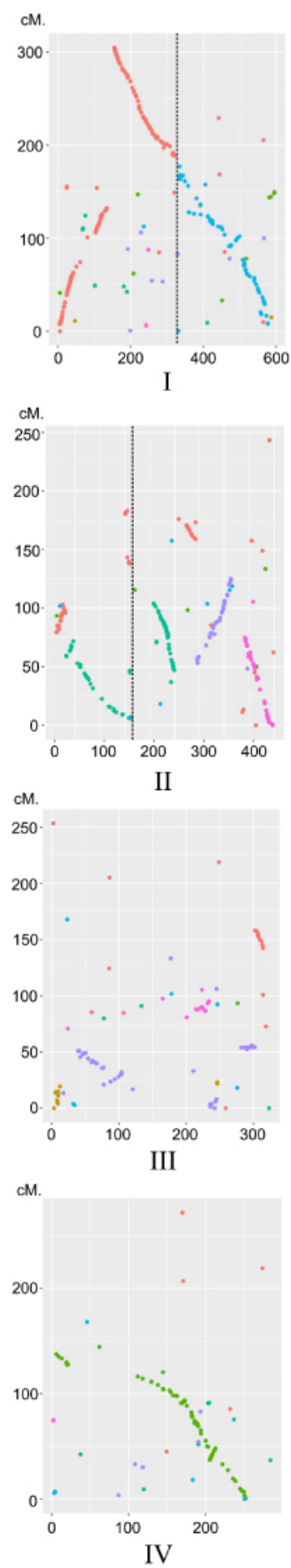

3D-DNA
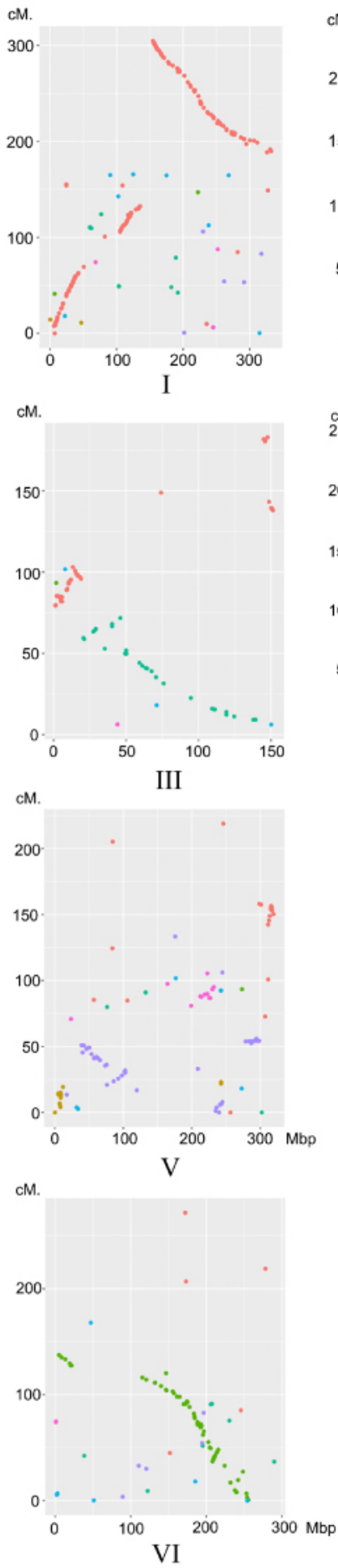
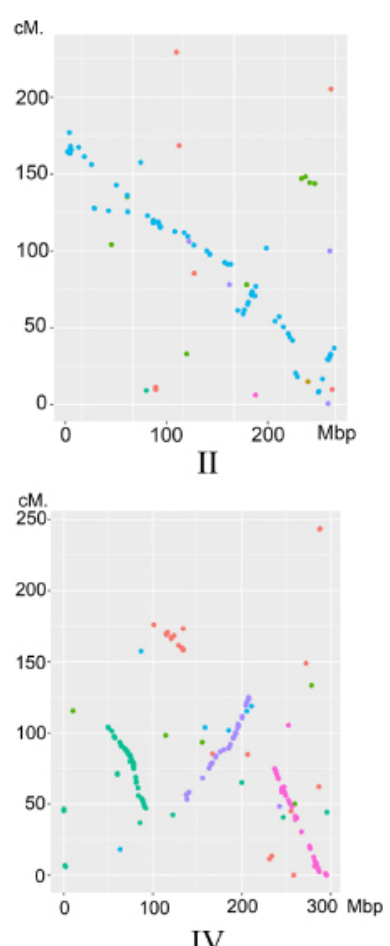

Linkage.group

- Vf_Chr-l

- Vf_Chr-1.2

- Vf_Chr-II

- Vf_Chr-III

- Vf_Chr-IV

- Vf_Chr-V

- Vf_Chr-VI

Figure 4. Comparison of HiRise and 3D-DNA-assembled pseudo-chromosomes to the Vicia faba genetic linkage map. Left, comparison of the four HiRise pseudo-chromosomes to the genetic linkage map; right, comparison of the six 3D-DNA pseudo-chromosomes to the linkage map. The $x$ axes present the coordinates of the pseudochromosomes, the $y$ axis presents the cumulative distance on the $V$. faba linkage map. Each color corresponds to a linkage group on the $V$. faba linkage map. 3D-DNA pseudo-chromosomes I and II are labelled as "a" and "b" in Figure 3, respectively. HiRise pseudo-chromosomes I, II, III and IV are labelled as "c", "d", "e" and "f” in Figure 3, respectively. 


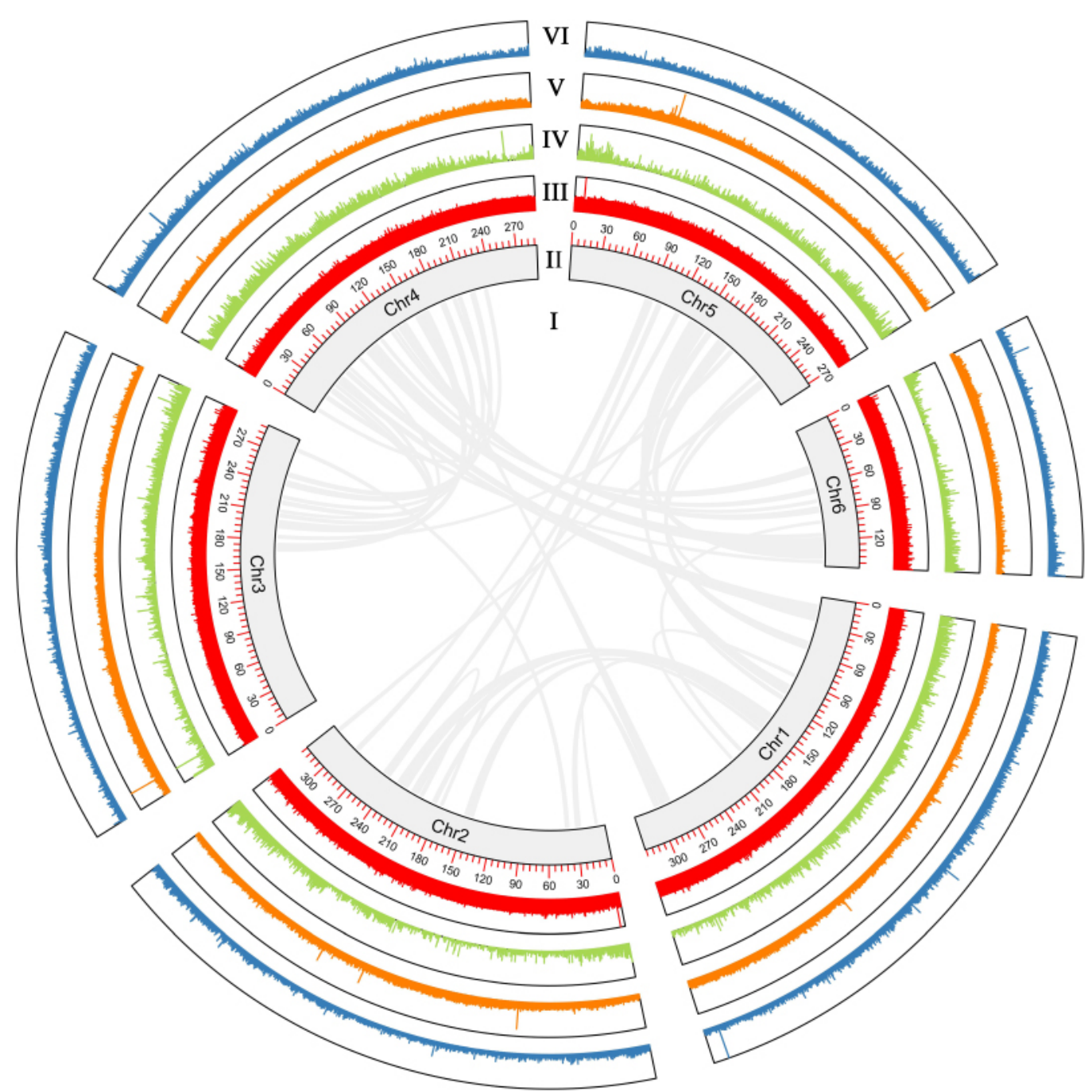

Figure 5. Circos plot showing the characterization of the Vicia sativa genome assembly. (I) Syntenic regions within the V. sativa genome based on homology searches using MCscan in Jcvi (MCScan, RRID:SCR_017650) [75] requiring $\geq 10$ genes per block (links). (II) Pseudo-chromosome length in Mbp. (III) GC content in non-overlapping $10 \mathrm{Kbp}$ windows (histograms). (IV) Gene density in non-overlapping 10-Kbp windows (histograms). (V) LTRtransposable element density in non-overlapping 10-Kbp windows. (VI) Mutator TIR transposon density in non-overlapping 10-Kbp windows (histograms). Percentage of GC content, gene density, and transposable element density were calculated relative to the highest value present in the genome. $\mathrm{Chr}=$ pseudo-chromosome.

RRID:SCR_018970) [32] and LTR_FINDER_parallel v1.2 [33] into LTR_retriever v2.9.0 (LTR_retriever, RRID:SCR_017623) [34], suggest that the genome reached a reference quality.

\section{Genome annotation}

To annotate the $V$. sativa genome assembly, we masked repeat regions of the genome, then mapped the RNA-seq data to the masked genome and performed gene prediction. First, the repeat families found in the $V$. sativa genome were identified de novo and classified using the software package EDTA v1.9.6 [35] with the sensitive model setting. EDTA integrated multiple programs, including LTR_FINDER (LTR_Finder, RRID:SCR_015247) [36] and 


\begin{tabular}{lc|}
\hline Table 5. Mapping results of Illumina paired-end reads with short insert sizes. & \\
\hline Parameters & Percentage (\%) \\
\hline Reads mapping rate & 99.7 \\
Genome coverage & 84.1 \\
Coverage at least 5× & 81.9 \\
Coverage at least 10× & 78.3 \\
Coverage at least 20× & 76.7 \\
\hline
\end{tabular}

\begin{tabular}{llccc}
\hline \multirow{2}{*}{$\begin{array}{l}\text { Table 6. Prediction of transposable element percentage in the Vicia sativa genome. } \\
\text { Number of elements }\end{array}$} & Number of elements & $\begin{array}{c}\text { Length of occupied } \\
\text { (bp) }\end{array}$ & \% of genome \\
Retroelements & & $1,361,823$ & $1,064,507,557$ & 64.4 \\
& LINEs & 5,620 & $2,743,407$ & 0.2 \\
DNA transposons & LTR elements & $1,356,203$ & $1,061,764,150$ & 64.2 \\
& Mutator TIR & 704,467 & $242,003,507$ & 14.6 \\
& transposon & 209,091 & $116,510,919$ & 7.0 \\
& hobo-Activator & & & 0.0 \\
Unclassified & Tourist/Harbinger & 88 & 34,340 & 0.01 \\
Simple repeats & & 318 & 212,845 & 4.2 \\
Low complexity & & 174,030 & $69,154,926$ & 0.6 \\
Total & & 29,826 & $10,230,793$ & 0.1 \\
\hline
\end{tabular}

\begin{tabular}{|c|c|c|c|c|c|c|}
\hline Gene set & $\begin{array}{c}\text { Number of } \\
\text { genes }\end{array}$ & $\begin{array}{l}\text { CDS + intron } \\
\text { length (avg.) }\end{array}$ & $\begin{array}{l}\text { CDS length } \\
\text { (avg.) }\end{array}$ & $\begin{array}{l}\text { Exon length } \\
\text { (avg.) }\end{array}$ & $\begin{array}{l}\text { Intron length } \\
\text { (avg.) }\end{array}$ & $\begin{array}{l}\text { Exons per gene } \\
\text { (avg.) }\end{array}$ \\
\hline Braker & 53,218 & 2267.11 & 956.97 & 223.43 & 415.13 & 4.42 \\
\hline
\end{tabular}

RepeatModeler (RepeatModeler, RRID:SCR_015027), which generated a non-redundant transposable element (TE) library used to annotate the TE regions on the genome. The TE library generated from EDTA was also used as an input to RepeatMasker v4.1.2 (RepeatMasker, RRID:SCR_012954) to identify and perform "hard-masking” and "soft-masking" for the repetitive region on the genome. A total of $83.9 \%$ of the genome was masked, and $64.4 \%$ of the genome was detected as LTR elements (Table 6).

After genome masking, a combination of $a b$ initio prediction and transcript evidence from the RNA-seq was used for gene prediction. Briefly, each RNA-seq data set was trimmed for low quality bases using TrimGalore v0.4.2, and mapped to the hard-masked-genome by using STAR v2.7.9 (STAR, RRID:SCR_004463) [37] to generate BAM files. Then the soft-masked genome and the BAM files generated from STAR were used for gene prediction using BRAKER v2.1.6 (BRAKER, RRID:SCR_018964) [38]. A total of 53,218 predicted protein-coding-genes were identified (Table 7). To assess the completeness of these protein-coding-genes, BUSCO v5.1.3 with fabales_odb10 gene sets were used which then identified 5127 (95.6\%) complete, 395 (7.4\%) duplicated, 70 fragmented (1.3\%) and 169 missing (3.1\%) orthologs.

Putative functions of the predicted protein-coding-genes were characterized by comparing the predicted proteins against the SwissProt and National Center for Biotechnology Information (NCBI) non-redundant database using Diamond v2.0.11 (DIAMOND, RRID:SCR_016071) [39] with e-value cut-off of $1 \times 10^{-5}$. Protein motifs and domains were annotated by comparing the predicted proteins against the InterPro 


\begin{tabular}{llcc}
\hline Table 8. Number of genes with homologs or functional classifications based on different databases. \\
\hline Database & & Annotated number & Annotated percentage (\%) \\
NCBI-NR & & 44,400 & 83.4 \\
Swiss-Prot & All & 31,071 & 58.4 \\
InterPro & Pfam & 43,549 & 81.8 \\
& GO & 30,264 & 56.9 \\
& Pfam & 8,983 & 16.9 \\
Eggnog & KEGG_pathway & 34,527 & 64.9 \\
& KEGG_ko & 10,777 & 20.3 \\
& GO & 16,898 & 31.8 \\
Annotated & & 17,987 & 33.8 \\
Total & & 47,580 & 89.4 \\
\hline
\end{tabular}

\begin{tabular}{|c|c|c|c|c|c|}
\hline Type & & Copy number & $\begin{array}{l}\text { Average length } \\
\text { (bp) }\end{array}$ & Total length (bp) & $\%$ of genome \\
\hline miRNA & & 158 & 111.3 & 17,579 & 0.001 \\
\hline tRNA & & 1382 & 73.7 & 101,891 & 0.006 \\
\hline \multirow[t]{4}{*}{ rRNA } & rRNA & 649 & 440.1 & 285,638 & 0.017 \\
\hline & $18 \mathrm{~S}$ & 32 & 1763.5 & 56,431 & 0.003 \\
\hline & $28 \mathrm{~S}$ & 39 & 4249.9 & 165,745 & 0.010 \\
\hline & $5 S$ & 578 & 109.8 & 63,462 & 0.003 \\
\hline \multirow[t]{4}{*}{ snRNA } & snRNA & 1777 & 107.5 & 191,047 & 0.011 \\
\hline & CD-box & 1551 & 102.4 & 158,835 & 0.010 \\
\hline & HACA-box & 69 & 126.7 & 8,740 & 0.001 \\
\hline & splicing & 157 & 149.5 & 23,472 & 0.001 \\
\hline
\end{tabular}

database using Interproscan v5.52-86.0 (InterProScan, RRID:SCR_005829) [40]. The predicted proteins were also assigned with Gene Ontology (GO) terms corresponding to the InterPro entries using Interproscan v5.52-86.0. In addition, we compared the predicted proteins against the EggNOG database v5.0 (eggnog, RRID:SCR_002456) [41] using eggNOG-mapper v2.1.4-2 (eggNOG-mapper, RRID:SCR_021165) [42] and assigned them with Kyoto Encyclopedia of Genes and Genomes (KEGG) pathways and KEGG orthologous groups (KO). As a result, we were able to annotate 47,580 (89.4\%) predicted protein-coding genes with at least one function term (Table 8).

In addition, we also identified and annotated non-coding RNA in the V. sativa genome. tRNA was identified using tRNAscan-SE v2.07 (tRNAscan-SE, RRID:SCR_010835) [43], rRNA was identified using Rnammer v1.2 (RNAmmer, RRID:SCR_017075) [44] and other types of non-coding RNA were identified by using Infernal v1.1.4 (Infernal, RRID:SCR_011809) [45] based on the Rfam database (Rfam, RRID:SCR_007891) [46]. Overall, 3966 of noncoding genes were annotated, including 158 miRNA, 649 rRNA and 1777 snRNA (Table 9).

\section{Phylogenetic tree construction and divergence time estimation}

We identified the orthogroups, phylogenetic positions and divergence times between $V$ sativa and 11 other plant species. The source of the protein-coding sequences used in our analysis are listed in Table 10. First, protein sequences of $V$. sativa, Pisum sativum, Medicago truncatula, Trifolium pratense, Phaseolus vulgaris, P. lunatus, Vigna unguiculata, Chamaecrista fasciculata, Faidherbia albida, Cercis canadensis, Carya illinoinensis, and Arabidopsis thaliana [47-54] were clustered into orthogroups using Orthofinder v2.5.4 


\begin{tabular}{|c|c|c|c|}
\hline Species & Abbreviation name & Source of data & Data version \\
\hline Vicia sativa & V. sat & This project & \\
\hline Pisum sativum & P. sat & URGI & V1a \\
\hline Medicago truncatula & M. tru & INRA & MtA17 r5 \\
\hline Trifolium pratense & T. pra & Phytozome & v2 \\
\hline Phaseolus vulgaris & P. vul & Phytozome & v2.1 \\
\hline Phaseolus lunatus & P. lun & Phytozome & v1 \\
\hline Vigna unguiculata & V. ung & Phytozome & v1.2 \\
\hline Chamaecrista fasciculata & C. fas & GigaDB & v1 \\
\hline Faidherbia albida & F. alb & GigaDB & N/A \\
\hline Cercis canadensis & C. can & GigaDB & v1 \\
\hline Carya illinoinensis & C. ill & Phytozome & v1.1 \\
\hline Arabidopsis thaliana & A. tha & Phytozome & TAIR10 \\
\hline
\end{tabular}

\begin{tabular}{|c|c|c|c|c|c|c|}
\hline Species & Number of genes & $\begin{array}{c}\text { Number of } \\
\text { orthogroups }\end{array}$ & $\begin{array}{l}\text { Number of genes in } \\
\text { orthogroups }\end{array}$ & $\begin{array}{c}\text { Number of } \\
\text { species-specific } \\
\text { orthogroups }\end{array}$ & $\begin{array}{l}\text { Number of genes in } \\
\text { species-specific } \\
\text { orthogroups }\end{array}$ & Single copy genes \\
\hline V. sat & 53,218 & 19,096 & 48,028 & 1774 & 8,594 & 10,009 \\
\hline P. sat & 57,835 & 19,012 & 51,576 & 2203 & 10,289 & 8,131 \\
\hline M. tru & 44,618 & 18,528 & 38,693 & 909 & 3,180 & 10,755 \\
\hline T. pra & 39,943 & 18,366 & 36,476 & 791 & 2,558 & 10,686 \\
\hline P. vul & 27,433 & 16,521 & 26,884 & 47 & 137 & 10,660 \\
\hline P. lun & 43,997 & 16,918 & 42,007 & 408 & 7,518 & 10,730 \\
\hline V. ung & 31,948 & 16,741 & 30,176 & 336 & 1,463 & 10,297 \\
\hline C. fas & 32,832 & 14,944 & 31,229 & 472 & 4,336 & 9,630 \\
\hline F. alb & 28,979 & 15,695 & 26,573 & 450 & 1,666 & 9,883 \\
\hline C. can & 34,023 & 16,165 & 32,407 & 694 & 3,767 & 12,289 \\
\hline C. ill & 31,911 & 15,424 & 30,007 & 528 & 2,501 & 7,830 \\
\hline A. tha & 27,416 & 14,171 & 24,887 & 870 & 4,286 & 8,851 \\
\hline
\end{tabular}

(OrthoFinder, RRID:SCR_017118) [55] with default parameters. A total of 10,009 single-copy and 43,209 multi-copy genes were identified in the $V$. sativa annotation (Figure 6B, Table 11), forming 19,096 orthogroups (Figure 6A, Table 10). Comparing orthogroups amongst $V$. sativa, $P$. sativum, $M$. truncatula, P. vulgaris, F. albida, we identified 2309 orthogroups that are specific to $V$. sativa (Figure 6A). Orthofinder was further used to perform phylogenetic reconstruction with the multiple sequence alignment approach (using the -msa command). The generated species tree has a support value of one on all nodes (Figure 7), indicating the high reliability of the revealed phylogenetic relationships.

To estimate divergence times between $V$. sativa and other important legume species (Table 10), coding sequences of 64 randomly selected single copy orthologs (see Supplementary File 1 [57]) were aligned using MACSE v1.2 [58]. Low-quality regions of each alignment were trimmed using Trimal v1.4.1 (trimAl, RRID:SCR_017334) [59], resulting in high-quality alignments amounting to $139,956 \mathrm{bp}$. Individual alignments were then imported into Beast v2.6.3 (BEAST2, RRID:SCR_017307) [60] for phylogenetic dating. Substitution models were selected using BEAST Model Test [61] for each alignment and were allowed to coalesce using unlinked relaxed log-normal molecular clocks [62]. A calibrated Yule prior [63] was used to inform tree building and speciation with four node calibrations (Table 12). First, a log normal distribution of 89.3 MYA (5\% quantile 97.9 million years ago [MYA], median 106 MYA, 95\% quantile 121 MYA) [64] was used to inform the root 
(A)

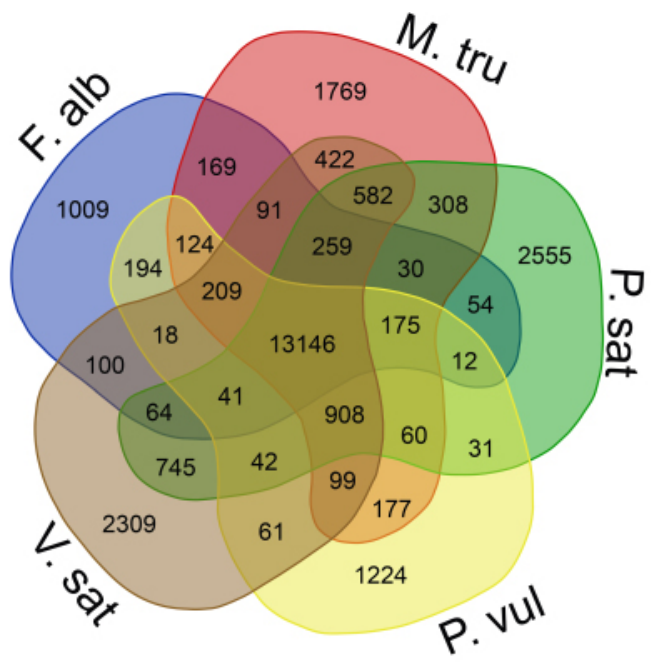

(C)

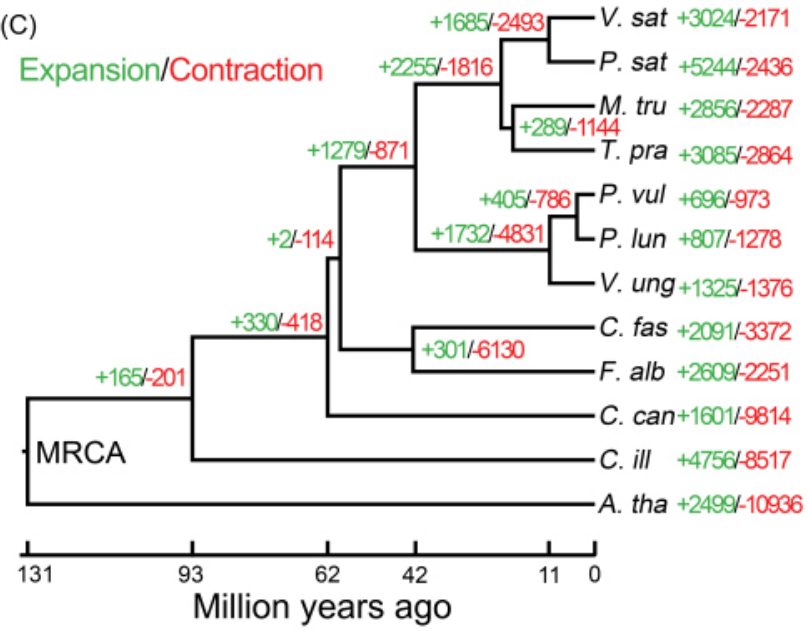

(B)
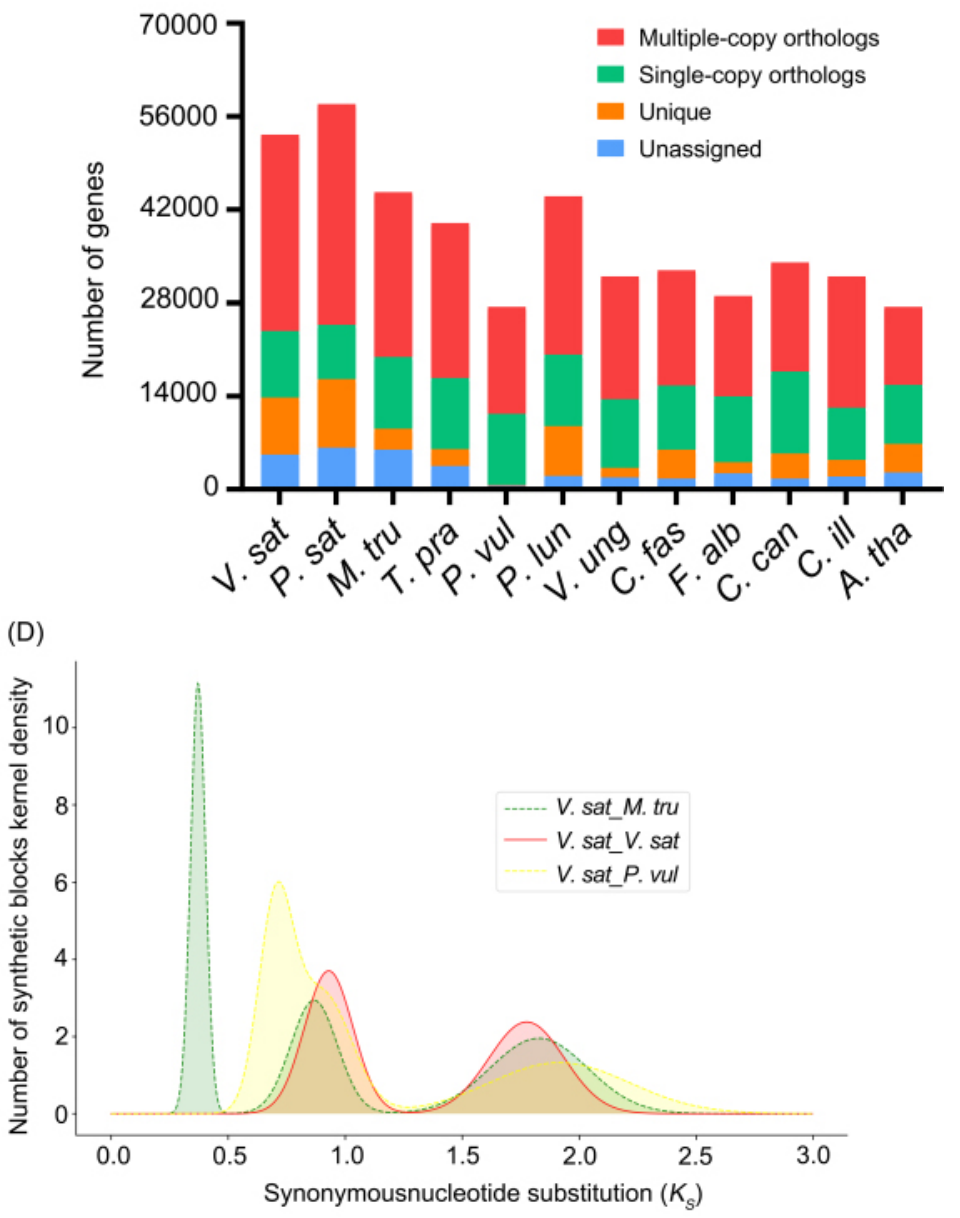

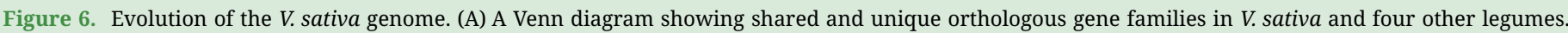

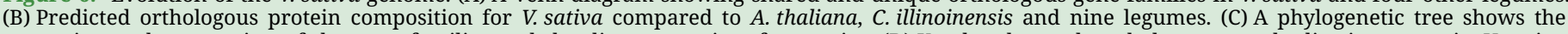

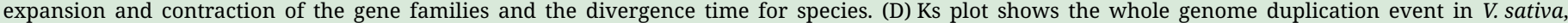

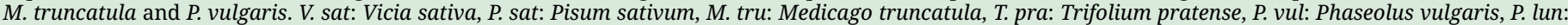

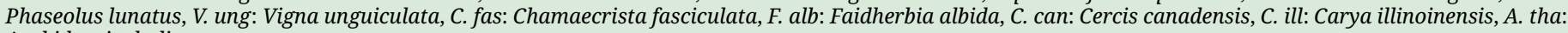
Arabidopsis thaliana.

prior (Brassicaceae, Fabaceae split). Three fossil calibrations were then set using CladeAge [65]: (i) Fabaceae (Figure 8 red dot; 65.3 MYA) [66], (ii) Caesalpinioideae (Figure 8 blue dot; 58 MYA) [67, 68], and (iii) Papilionoideae (Figure 8 green dot; 55 MYA) [69].

Furthermore, a net diversification rate was set to $0.1-0.134$ to construct a distribution around the literature value of 0.117 [70], turnover rate was set to $0.823-0.883$ to construct a distribution around the literature value of 0.853 [70], and sampling rate of 0.000034-0.013 [71] was set to determine CladeAge prior distributions. Final chain length of the Markov Chain Monte Carlo (MCMC; 600 million) was determined through continuous examination of the log file using Tracer until proper mixing was observed. This allowed us to determine a robust estimate for the most common recent ancestor (MRCA) of $V$. sativa and P. sativum at 10.6 (95\% Highest Posterior Density: 9.9-11.4) MYA (Figure 8). Gene family 


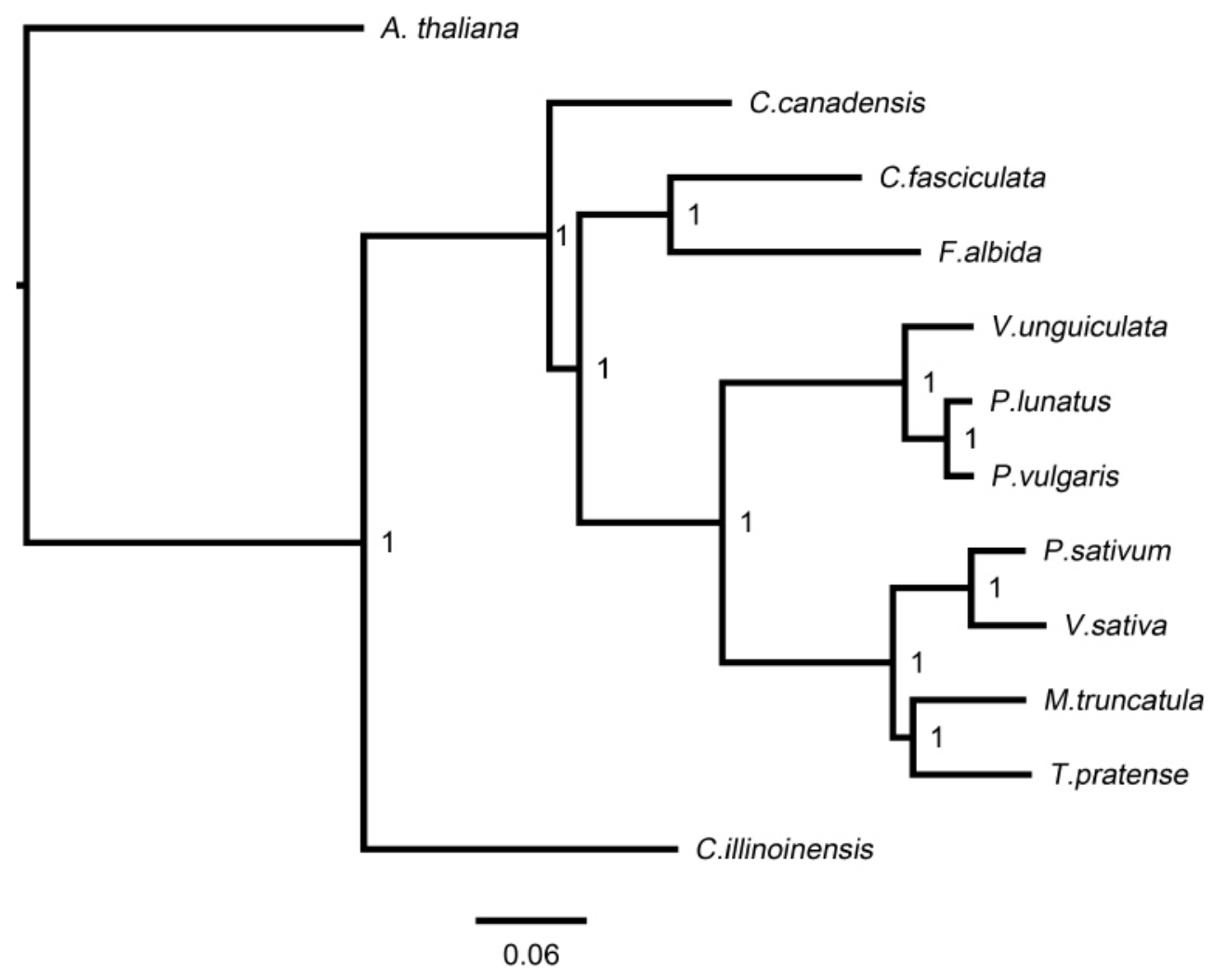

Figure 7. Species tree generated by Orthofinder using a multiple sequence alignment approach. Node label shows the Shimodaira-Hasegawa test supporting value [56].

\begin{tabular}{|c|c|c|c|}
\hline Node & Definition & Fossil & Age (Ma) \\
\hline Yellow & SG Brassicales & $\begin{array}{l}\text { Flowers of Dressiantha } \\
\text { bicarpellate; USA }\end{array}$ & 89.3 \\
\hline Red & SG Leguminosae & Seedpods and leaflets; USA & 65.3 \\
\hline Blue & SG Caesalpinioideae & Bipinnate leaves; Colombia & 58 \\
\hline Green & SG Papilionoideae & $\begin{array}{l}\text { Flowers of Barnebyanthus } \\
\text { buchananensis; USA }\end{array}$ & 55 \\
\hline
\end{tabular}

expansion and contraction analysis using CAFE v4.2.1 (Computational Analysis of gene Family Evolution, RRID:SCR_018924) [72] with a single $\lambda$ revealed 5195 gene families that have undergone gene expansion (3024) or contraction (2171) since the MRCA of V. sativa and $A$. thaliana (Figure 6C).

To identify whole genome duplication events (WGD), WGDI v0.5.1 [73] was used to identify gene collinearity between $V$. sativa, $M$. truncatula and $P$. vulgaris. The $K_{s}$ (synonymous substitutions per synonymous site) value was calculated based on the identified collinearity gene to construct a frequency distribution map. The Ks distribution indicated that $V$. sativa, $M$. truncatula and P. vulgaris share the same ancestral WGD event. The estimated time of this WGD event ( $\sim 58$ MYA) [74] and the corresponding Ks value ( $\sim 0.93$, Figure $6 \mathrm{D}$ ) reveal that the average mutation rate of $V$. sativa genome is $8.02 \times 10^{-9}$ per site per year. 


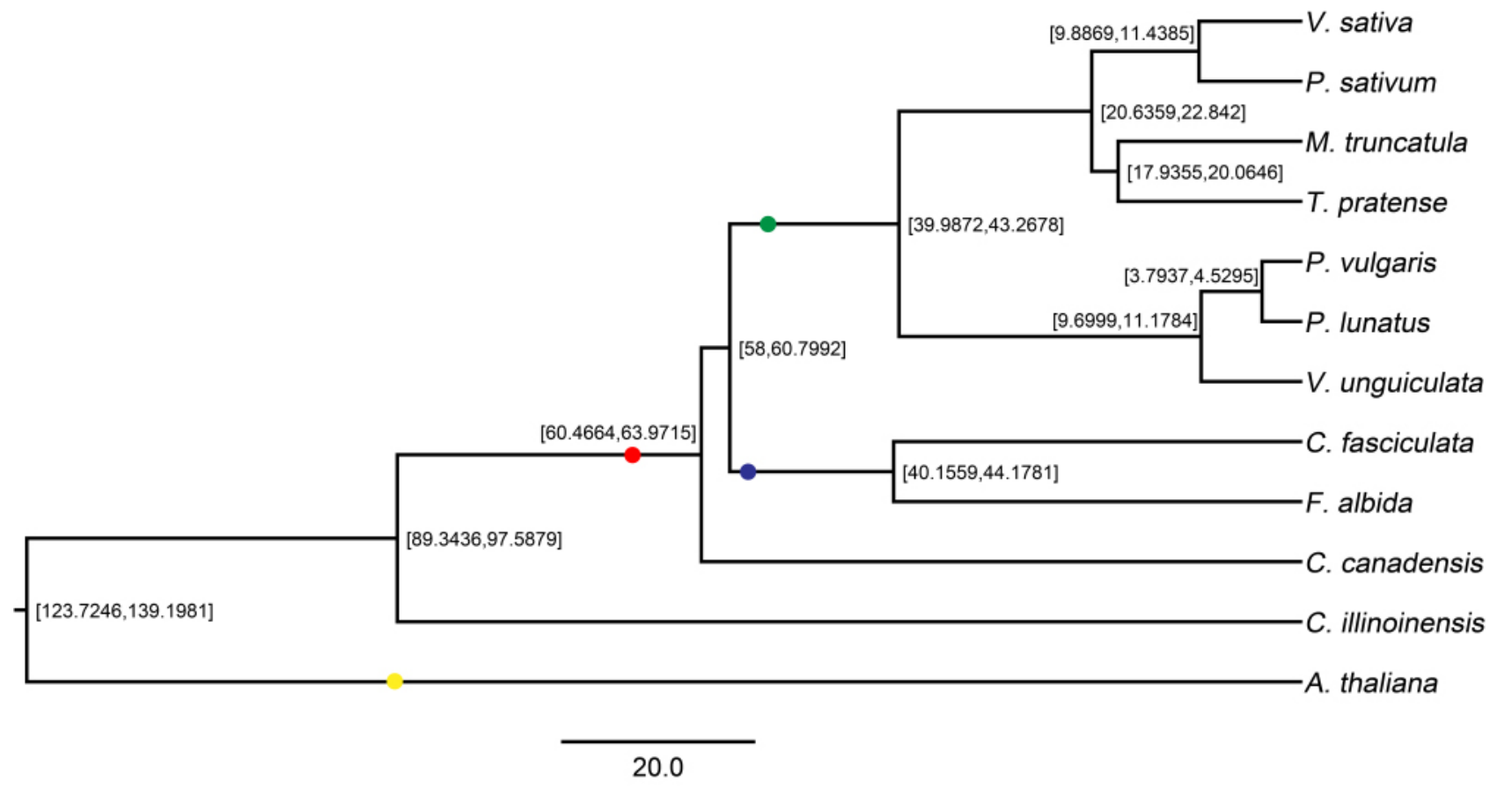

Figure 8. Divergence time estimation for Vicia sativa and other species. The node labels show the 95\% Highest Posterior Density of species divergence time. Units shown on the scale bar are million years ago. Yellow, red, blue and green dots correspond to fossil calibration points.

\section{REUSE POTENTIAL}

Understanding the genetic, epigenetic and epitranscriptomic basis of the evolutionary processes shaping drought tolerance, low nutrient requirements and adaption to broad habitats requires comparison of multiple legume genomes, preferentially assembled at the chromosome level. In this study, we present a complete chromosome-level genome assembly for the legume $V$. sativa (common vetch) and provided a detailed genome annotation. There are $>19,000$ species of legumes, about 200 within the Vicia genus, and this genome will serve as an excellent reference for the assembly of other Vicia genomes. The $V$. sativa genome will also facilitate comparative analyses aimed at understanding the evolutionary origin and dynamics of legume specific gene families. Our new $V$. sativa genome will greatly benefit legume researchers and plant breeders who are interested in conventional as well as engineering crop improvement.

\section{DATA AVAILABILITY}

Final assembly and original Nanopore assembly, as well as annotation files, Supplementary File 1, predicted transcript and protein sequences, and bioinformatics supporting information, were deposited in the database GigaDB [57]. Additionally, assembly, Illumina and Nanopore subreads, and transcriptome raw data are available at NCBI and can be accessed with BioProject PRJNA762450 and BioSample SAMN21393724. Illumina and Nanopore subreads can be obtained, with SRR16004114 and SRR16004115; and RNA-sequencing raw reads, as follows: SAMN21545804, SAMN21545805, SAMN21545806, SAMN21545807 and SAMN21545808. Additional data is available in the GigaScience GigaDB database [57]. 


\section{DECLARATIONS}

\section{List of abbreviations}

CHiCAGO: Capture Hi-C Analysis of Genomic Organisation; Gbp: gigabase pairs; Kbp: kilobase pairs; Mbp: megabase pairs; MCRA: most common recent ancestor; MYA: million years ago; NCBI: National Center for Biotechnology Information; TE: transposable element; WGS: whole genome sequencing, WGD: whole-genome duplication.

\section{ETHICAL APPROVAL}

Not applicable.

\section{CONSENT FOR PUBLICATION}

Not applicable.

\section{COMPETING INTERESTS}

The authors declare that they have no competing interests.

\section{FUNDING}

This work was funded by the Department of Industry, Science, Energy and Resources (grant number ACSRF 48187), the National Natural Science Foundation of China (grant number 31722055) and an Australia Research Council Future Fellowship (grant number FT130100525) awarded to IRS. HX, CW and VN were supported by University of Adelaide Research Training Scholarships (RTS) and University research support. VN was also supported by an AW Howard Memorial Trust Postgraduate Research fellowship.

\section{AUTHORS' CONTRIBUTIONS}

HW conducted the genome assembly, genome analysis and wrote the manuscript. VN prepared DNA and RNA for sequencing and co-wrote the manuscript. CW assisted with the genome assembly. IRS conceived and managed the project, interpreted the data and drafted the figures. ZL interpreted the data. All authors read, edited, revised, and approved the manuscript final version.

\section{ACKNOWLEDGEMENTS}

We are grateful to the high-performance computing infrastructure provided by the University of Adelaide. We thank the Australian National Vetch Breeding program for gifting the $V$. sativa seed.

\section{REFERENCES}

1 Navrátilová A, Neumann P, Macas J, Karyotype analysis of four Vicia species using in situ hybridization with repetitive sequences. Ann. Bot., 2003; 91(7): 921-926.

2 Hanelt P, Mettin D, Biosystematics of the genus Vicia L. (Leguminosae). Annu. Rev. Ecol. Syst., 1989; 20(1): 199-223.

3 Çceliktas N, Can E, Hatipoğlu R et al. Comparison between a wild population and cultivar of common vetch (Vicia sativa L., Fabaceae) on cytological and agronomic characteristics. New Zealand J. Agric. Res., 2006; 49: 389-393.

4 Zeven AC, Zhukovsky PM, Dictionary of Cultivated Plants and their Centres of Diversity: Excluding Ornamentals, Forest Trees and Lower Plants. Wageningen: Pudoc, 1975.

5 Mao Z, Fu H, Nan Z et al. Fatty acid, amino acid, and mineral composition of four common vetch seeds on Qinghai-Tibetan plateau. Food Chem., 2015; 171: 13-18. 
6 Pfeffer M, Ressler C, $\beta$-cyanoalanine, an inhibitor of rat liver cystathionase. Biochem. Pharmacol., 1967; 16(12): 2299-2308.

7 Meydani JA, Rahmati M, Karimi E et al. Dryland soil water storage susceptibility to different soil tillage practices under Vetch-Wheat crop rotation. Azarian J. Agric., 2014; 1: 18-24.

8 Mueller T, Thorup-Kristensen $\mathbf{K}$, N-fixation of selected green manure plants in an organic crop rotation. Biol. Agric. Hort., 2001; 18(4): 345-363.

9 Tenopala J, González FJ, de la Barrera E, Physiological responses of the green manure, Vicia sativa, to drought. Bot. Sci., 2012; 90(3): 263-285.

10 Nguyen V, Riley S, Nagel S et al. Common Vetch: a drought tolerant, high protein neglected leguminous crop with potential as a sustainable food source. Front. Plant Sci., 2020; 11: 818.

11 Jia C, Dong D, Zhou Q et al. Significant cell differences in pod ventral suture in shatter-resistant and shatter-susceptible common vetch accessions. Crop Sci., 2021; 61(3): 1749-1759.

12 Dong R, Dong D, Luo D et al. Transcriptome analyses reveal candidate pod shattering-associated genes involved in the pod ventral sutures of common vetch (Vicia sativa L.). Front. Plant Sci., 2017; 8: 649.

13 De la Rosa L, Zambrana E, Ramirez-Parra E, Molecular bases for drought tolerance in common vetch: designing new molecular breeding tools. BMC Plant Biol., 2020; 20(1): 1-18.

14 Sambrook J, Russell DW, Purification of nucleic acids by extraction with phenol: chloroform. Cold Spring Harb. Protoc., 2006; 2006(1): pdb.prot4455. https://dx.doi.org/10.1101/pdb.prot4455.

15 Biosciences P, Guidelines for using a salt: chloroform wash to clean up gDNA. 2019; https://www.pacb.com/wp-content/uploads/2015/09/Shared-Protocol-Guidelines-for-Using-a-SaltChloroform-Wash-to-Clean-Up-gDNA.pdf.

16 Putnam NH, O’Connell BL, Stites JC et al. Chromosome-scale shotgun assembly using an in vitro method for long-range linkage. Genome Res., 2016; 26(3): 342-350.

17 Lieberman-Aiden E, Van Berkum NL, Williams L et al. Comprehensive mapping of long-range interactions reveals folding principles of the human genome. Science, 2009; 326(5950): 289-293.

18 David R, Burgess A, Parker B et al. Transcriptome-wide mapping of RNA 5-methylcytosine in Arabidopsis mRNAs and noncoding RNAs. Plant Cell, 2017; 29(3): 445-460.

19 Marçais G, Kingsford C, A fast, lock-free approach for efficient parallel counting of occurrences of k-mers. Bioinformatics, 2011; 27(6): 764-770.

20 Vurture GW, Sedlazeck FJ, Nattestad M et al. GenomeScope: fast reference-free genome profiling from short reads. Bioinformatics, 2017; 33(14): 2202-2204.

21 Macas J, Novak P, Pellicer J et al. In depth characterization of repetitive DNA in 23 plant genomes reveals sources of genome size variation in the legume tribe Fabeae. PLoS One, 2015; 10(11): e0143424.

22 Koren S, Walenz BP, Berlin $\mathbf{K}$ et al. Canu: scalable and accurate long-read assembly via adaptive k-mer weighting and repeat separation. Genome Res., 2017; 27(5): 722-736.

23 Walker BJ, Abeel T, Shea T et al. Pilon: an integrated tool for comprehensive microbial variant detection and genome assembly improvement. PLoS One, 2014; 9(11): e112963.

24 Simão FA, Waterhouse RM, Ioannidis P et al. BUSCO: assessing genome assembly and annotation completeness with single-copy orthologs. Bioinformatics, 2015; 31(19): 3210-3212.

25 Van Berkum NL, Lieberman-Aiden E, Williams L et al. Hi-C: a method to study the three-dimensional architecture of genomes. JoVE, 2010; 39: e1869.

26 Cairns J, Freire-Pritchett P, Wingett SW et al. CHiCAGO: robust detection of DNA looping interactions in Capture Hi-C data. Genome Biol., 2016; 17(1): 1-17.

27 Dudchenko O, Batra SS, Omer AD et al. De novo assembly of the Aedes aegypti genome using Hi-C yields chromosome-length scaffolds. Science, 2017; 356(6333): 92-95.

28 Durand NC, Shamim MS, Machol I et al. Juicer provides a one-click system for analyzing loop-resolution Hi-C experiments. Cell Syst., 2016; 3(1): 95-98.

29 Marçais G, Delcher AL, Phillippy AM et al. MUMmer4: a fast and versatile genome alignment system. PLoS Comp Biol., 2018; 14(1): e1005944.

30 Sudheesh S, Kimber R, Braich S et al. Construction of an integrated genetic linkage map and detection of quantitative trait loci for ascochyta blight resistance in faba bean (Vicia faba L.). Euphytica, 2019; 215(3): 42. 
31 Guan D, McCarthy SA, Wood J et al. Identifying and removing haplotypic duplication in primary genome assemblies. Bioinformatics, 2020; 36(9): 2896-2898.

32 Ellinghaus D, Kurtz S, Willhoeft U, LTRharvest, an efficient and flexible software for de novo detection of LTR retrotransposons. BMC Bioinform., 2008; 9(1): 1-14.

33 Ou S, Jiang N, LTR_FINDER_parallel: parallelization of LTR_FINDER enabling rapid identification of long terminal repeat retrotransposons. Mobile DNA, 2019; 10(1): 1-3.

34 Ou S, Jiang N, LTR_retriever: a highly accurate and sensitive program for identification of long terminal repeat retrotransposons. Plant Physiol., 2018; 176(2): 1410-1422.

35 Ou S, Su W, Liao Y et al. Benchmarking transposable element annotation methods for creation of a streamlined, comprehensive pipeline. Genome Biol., 2019; 20(1): 1-18.

36 Xu Z, Wang H, LTR_FINDER: an efficient tool for the prediction of full-length LTR retrotransposons. Nucleic Acids Res., 2007; 35(suppl_2): W265-W268.

37 Dobin A, Davis CA, Schlesinger F et al. STAR: ultrafast universal RNA-seq aligner. Bioinformatics, 2013; 29(1): 15-21.

38 Brůna T, Hoff KJ, Lomsadze A et al. BRAKER2: Automatic eukaryotic genome annotation with GeneMark-EP+ and AUGUSTUS supported by a protein database. NAR Genom. Bioinform., 2021; 3(1): lqaa108.

39 Buchfink B, Xie C, Huson Dh, Fast and sensitive protein alignment using DIAMOND. Nat. Meth., 2015; 12(1): 59-60.

40 Jones P, Binns D, Chang H-Y et al. InterProScan 5: genome-scale protein function classification. Bioinformatics, 2014; 30(9): 1236-1240.

41 Huerta-Cepas J, Szklarczyk D, Heller D et al. eggNOG 5.0: a hierarchical, functionally and phylogenetically annotated orthology resource based on 5090 organisms and 2502 viruses. Nucleic Acids Res., 2019; 47(D1): D309-D314.

42 Cantalapiedra CP, Hernández-Plaza A, Letunic I et al. eggNOG-mapper v2: Functional Annotation, Orthology Assignments, and Domain Prediction at the Metagenomic Scale. Mol. Biol. Evol., 2021; 38: 5825-5829.

43 Schattner P, Brooks AN, Lowe TM, The tRNAscan-SE, snoscan and snoGPS web servers for the detection of tRNAs and snoRNAs. Nucleic Acids Res., 2005; 33(suppl_2): W686-W689.

44 Lagesen K, Hallin P, Rødland EA et al. RNAmmer: consistent and rapid annotation of ribosomal RNA genes. Nucleic Acids Res., 2007; 35(9): 3100-3108.

45 Nawrocki EP, Eddy SR, Infernal 1.1: 100-fold faster RNA homology searches. Bioinformatics, 2013; 29(22): 2933-2935.

46 Kalvari I, Argasinska J, Quinones-Olvera N et al. Rfam 13.0: shifting to a genome-centric resource for non-coding RNA families. Nucleic Acids Res., 2018; 46(D1): D335-D342.

47 Pecrix Y, Staton SE, Sallet E et al. Whole-genome landscape of Medicago truncatula symbiotic genes. Nat. Plants, 2018; 4(12): 1017-1025.

48 De Vega JJ, Ayling S, Hegarty $\mathbf{M}$ et al. Red clover (Trifolium pratense L.) draft genome provides a platform for trait improvement. Sci. Rep., 2015; 5(1): 1-10.

49 Lonardi S, Muñoz-Amatriaín M, Liang Q et al. The genome of cowpea (Vigna unguiculata [L.] Walp.). Plant J., 2019; 98(5): 767-782.

50 US Department of Energy Office of Science. DA-NIFA D-Ja: Phytozome 13. http://phytozome.jgi.doe.gov/.

51 Lovell JT, Bentley NB, Bhattarai G et al. Four chromosome scale genomes and a pan-genome annotation to accelerate pecan tree breeding. Nat. Commun., 2021; 12(1): 1-12.

52 Lamesch P, Berardini TZ, Li D et al. The Arabidopsis Information Resource (TAIR): improved gene annotation and new tools. Nucleic Acids Res., 2012; 40(D1): D1202-D1210.

53 Griesmann M, Chang Y, Liu X et al. Phylogenomics reveals multiple losses of nitrogen-fixing root nodule symbiosis. Science, 2018; 361(6398): eaat1743.

54 Chang Y, Liu H, Liu M et al. The draft genomes of five agriculturally important African orphan crops. GigaScience, 2019; 8(3): giy152. doi:10.1093/gigascience/giy152. 
55 Emms DM, Kelly S, OrthoFinder: phylogenetic orthology inference for comparative genomics. Genome Biol., 2019; 20(1): 1-14.

56 Shimodaira $\mathbf{H}$, An approximately unbiased test of phylogenetic tree selection. Syst. Biol., 2002; 51(3): 492-508.

57 Xi H, Nguyen V, Ward C et al. Supporting data for "Chromosome-level assembly of the common vetch reference genome (Vicia sativa)". GigaScience Database. 2022; http://dx.doi.org/10.5524/100954.

58 Ranwez V, Harispe S, Delsuc F et al. MACSE: Multiple Alignment of Coding SEquences accounting for frameshifts and stop codons. PLoS One, 2011; 6(9): e22594.

59 Capella-Gutiérrez S, Silla-Martínez JM, Gabaldón T, trimAl: a tool for automated alignment trimming in large-scale phylogenetic analyses. Bioinformatics, 2009; 25(15): 1972-1973.

60 Bouckaert R, Vaughan TG, Barido-Sottani J et al. BEAST 2.5: an advanced software platform for Bayesian evolutionary analysis. PLoS Comp. Biol., 2019; 15(4): e1006650.

61 Bouckaert RR, Drummond AJ, bModelTest: Bayesian phylogenetic site model averaging and model comparison. BMC Evol. Biol., 2017; 17(1): 1-11.

62 Drummond AJ, Ho SYW, Phillips MJ et al. Relaxed phylogenetics and dating with confidence. PLoS Biol., 2006; 4(5): e88.

63 Heled J, Drummond AJ, Calibrated tree priors for relaxed phylogenetics and divergence time estimation. Syst. Biol., 2012; 61(1): 138-149.

64 Magallón S, Gómez-Acevedo S, Sánchez-Reyes LL et al. A metacalibrated time-tree documents the early rise of flowering plant phylogenetic diversity. New Phytol., 2015; 207(2): 437-453.

65 Matschiner M, Musilová Z, Barth JM et al. Bayesian phylogenetic estimation of clade ages supports trans-Atlantic dispersal of cichlid fishes. Syst. Biol., 2017; 66(1): 3-22.

66 Lyson T, Miller I, Bercovici A et al. Exceptional continental record of biotic recovery after the Cretaceous-Paleogene mass extinction. Science, 2019; 366(6468): 977-983.

67 Koenen EJ, Ojeda DI, Bakker FT et al. The origin of the legumes is a complex paleopolyploid phylogenomic tangle closely associated with the cretaceous-paleogene (K-Pg) mass extinction event. Syst. Biol., 2021; 70(3): 508-526.

68 Wing SL, Herrera F, Jaramillo CA et al. Late Paleocene fossils from the Cerrejón Formation, Colombia, are the earliest record of Neotropical rainforest. Proc. Natl. Acad. Sci. USA, 2009; 106(44): 18627-18632.

69 Lavin M, Herendeen PS, Wojciechowski MF, Evolutionary rates analysis of Leguminosae implicates a rapid diversification of lineages during the tertiary. Syst. Biol., 2005; 54(4): 575-594.

70 Fiz-Palacios O, Schneider H, Heinrichs J et al. Diversification of land plants: insights from a family-level phylogenetic analysis. BMC Evol. Biol., 2011; 11(1): 1-10.

71 Silvestro D, Bacon CD, Ding W et al. Fossil data support a pre-Cretaceous origin of flowering plants. Nat. Ecol. Evol., 2021; 5(4): 449-457.

72 De Bie T, Cristianini N, Demuth JP et al. CAFE: a computational tool for the study of gene family evolution. Bioinformatics, 2006; 22(10): 1269-1271.

73 Sun P, Jiao B, Yang Y et al. WGDI: A user-friendly toolkit for evolutionary analyses of whole-genome duplications and ancestral karyotypes. bioRxiv. 2021; https://doi.org/10.1101/2021.04.29.441969.

74 Pfeil B, Schlueter J, Shoemaker R et al. Placing paleopolyploidy in relation to taxon divergence: a phylogenetic analysis in legumes using 39 gene families. Syst. Biol., 2005; 54(3): 441-454.

75 Tang H, Bowers JE, Wang X et al. Synteny and collinearity in plant genomes. Science, 2008; 320(5875): 486-488. 